\title{
Information Status
}

National Cancer Institute

\section{Source}

National Cancer Institute. Information Status. NCI Thesaurus. Code C70728.

A state of the information pertaining to the entity associated with a clinical study protocol indicating whether this information is active or inactive for the purpose of inclusion in the protocol planned activities. 